\title{
Consistency in Marked Digraphs
}

\author{
LOWELL W. BeINEKE \\ Department of Mathematics, Purdue University, Fort Wayne, Indiana 46805 \\ AND \\ Frank Harary \\ Department of Mathematics, University of Michigan, Ann Arbor, Michigan 48109
}

In this paper we strive towards a mathematical theory for "marked digraphs" in which the nodes are signed. For completeness, we begin with an extensive list of definitions, including that of "consistency" in marked digraphs. We then provide three different descriptions of the concept: one an alternative in terms of directed cycles, another in terms of partitioning the nodes, and a third in terms of arc-digraphs and balance. We conclude with two additional observations, one characterizing the structure of consistent strongly connected marked tournaments and the other giving a criterion for a digraph to be "markable" in a consistent way.

\section{Signed Digraphs and BaLAnce}

Balance theory in the study of two-valued (positive-negative) relations provides a mathematical model for "likes-dislikes" situations. It was introduced by Heider (1946) in the three-element $(P-O-X)$ setting. Cartwright and Harary (1956) extended the concept to directed graphs in general, and additional results on duality and measurement have been obtained by Harary $(1957,1959)$. Some of the configurations studied by these authors were directed graphs in which each arc is positive (for "likes") or negative (for "dislikes") and in which the nodes (points, vertices) represent people or objects. Figure 1 shows an example of such a structure. See Harary, Norman, and

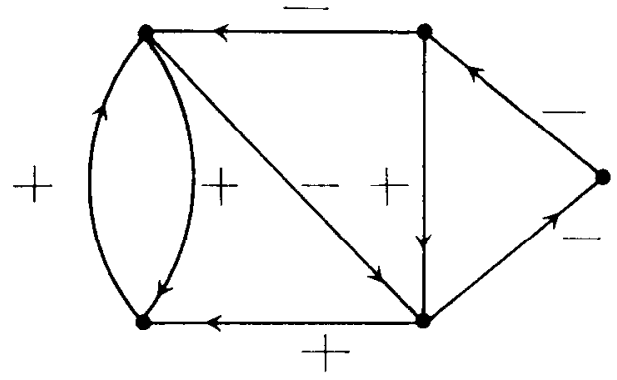

FIG. 1. A signed digraph. 
Cartwright (1965, Chapter 9), for an exposition of balance theory and for all concepts concerning digraphs not explicitly defined here.

A signed digraph is called balanced if each of its semicycles is positive. (A semicycle is a directed cycle in which some of its arcs have been reversed; its sign is the usual product of the signs of its arcs.) Cartwright et al. (1956) found the following criterion for balance.

Structural Theorem ror Balance. A signed digraph is balanced if and only if its nodes can be partitioned into two sets (one possibly empty) in such a way that arcs joining two nodes in the same set are positive and arcs joining two nodes in different sets are negative.

Zajonc (1968) observed that signed digraphs constitute the basic underlying structure of cognitive consistency. However, Osgood and Tannenbaum (1955) assign the signs to elements (standing for persons) rather than to the relationships (the arcs).

One can give an example of a simple situation in which signed nodes are important. Consider a group of people, each of whom either always lies or always tells the truth. Within the group there is a communication network of "who talks to whom." If a rumor or message is planted within the group, can any results be stated regarding the consistency of the versions heard by various group members?

In mathematical terms, the underlying structure for this situation is a directed graph in which the nodes have signs (positive or negative) and the arcs represent direct communication. The problem becomes that of determining the consistency of sign of the directed paths from one node to another. This is the topic to which we address oursclves in this paper.

\section{Marked DigRaphs AND Consistency}

A digraph (directed graph) consists of a set $V$ of nodes and a set $S$ of ordered pairs of nodes called arcs. We generally denote nodes by letters such as $u$, $v$, or $w$, and arcs by letters $a$ or $b$; an arc such as $(u, v)$ we frequently denote by $u v$. A diwalk consists of an alternating sequence of nodes and arcs $v_{0}, a_{1}, v_{1}, \ldots, v_{n-1}, a_{n}, v_{n}$ with arc $a_{i}$ directed from $v_{i-1}$ to $v_{i}$. The length of such a diwalk is $n$, the number of occurrences of arcs, and it is called a $v_{0}: v_{n}$ diwalk. A diwalk is closed if the first and last nodes are the same. A dipath is a diwalk in which all nodes are different, while a dicycle is a closed diwalk in which all nodes except the first and last are different. A digraph is called strongly connected (or strong) if there are dipaths from each node to all others. We are primarily interested in strong digraphs here.

A digraph is called marked if each node is designated as being either positive or negative; it is then denoted $M$. The sign of a diwalk in $M$ is defined as the product of the signs of all its nodes except the last. Finally, a marked digraph $M$ is called consistent if there do not exist two nodes $u$ and $v$ with diwalks from $u$ to $v$ of opposite signs. Figure 2 shows two marked digraphs; the first is consistent, the second is not (since there are both positive and negative diwalks from $u$ to $v$ ). 

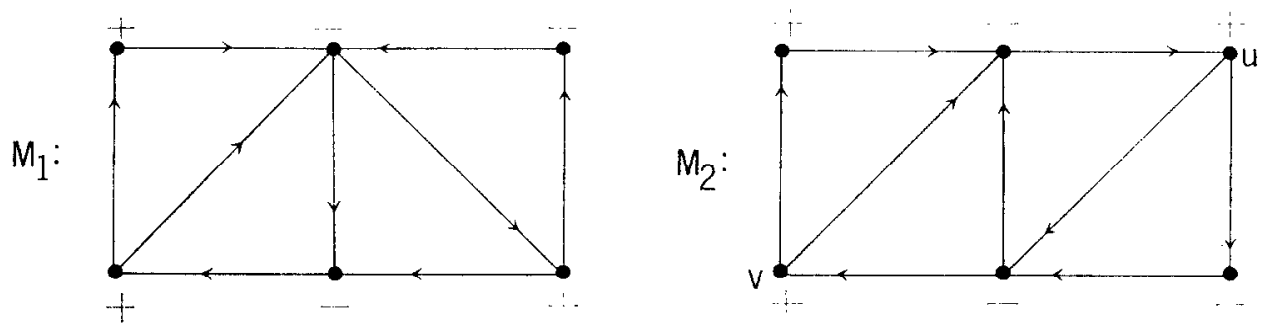

FIG. 2. Two marked digraphs, one consistent and one not.

We observe that in a strong marked digraph $M$, if for some pair of nodes $u$ and $v$ all $u: v$ diwalks have the same sign, then the entire structure $M$ is consistent. For, suppose this is not the case; that is, there are nodes $x$ and $y$ with a positive diwalk $W_{0}$ and a negative diwalk $W_{1}$ both from $x$ to $y$. Since the digraph is strong, there are dipaths $P$ from $u$ to $x$ and $Q$ from $y$ to $v$. It follows that there are $u: v$ diwalks of opposite sign, one formed as $P, W_{0}, Q$ and the other as $P, W_{1}, Q$.

Our first theorem simplifies the testing of strong marked digraphs for consistency by showing that one need not consider all diwalks, only dicycles.

THEOREM 1. The following statements are equivalent for a strongly connected marked digraph $M$ :

(1) Mis consistent.

(2) Every closed diwalk of $M$ is positive.

(3) Every dicycle of $M$ is positive.

Proof. (1) implies (2). This is proved by contradiction. Assume (1) holds but that $M$ has a negative closed diwalk $W$. Let $u$ be a negative node in $W$, and without loss of generality we may assume $u$ is the first node. Let $v$ denote the second node. Then the arc $u v$ provides a negative $u: v$ diwalk, while $W$ followed by $u v$ gives a positive one. This, however, contradicts (1) and proves the implication.

(2) implies (1). We again use a proof by contradiction. Assume that (2) holds but there are a positive diwalk $W_{0}$ and a negative diwalk $W_{1}$ both from node $u$ to node $v$. Let $W$ be any diwalk from $v$ to $u$. Then, depending on the sign of $W$, either the closed diwalk formed by $W$ and $W_{0}$ or that formed by $W$ and $W_{1}$ is negative. Hence (2) could not hold.

(2) implies (3). A fortiori if every closed diwalk is positive, so is every dicycle.

(3) implies (2). To prove this, we show that every negative closed diwalk contains the nodes and arcs of a negative dicycle. This we prove by induction on the number $n$ of arcs in the closed diwalk. The statement is true if $n=2$. Assume it is true for all values of $n$ less than $k$, and let $W$ be a closed negative diwalk of length $k$. If $W$ is not a dicycle, it can be considered as the succession of two closed diwalks of shorter length. One of 
these must be negative and, by the induction hypothesis, contains a negative dicycle. So by the Principle of Mathematical Induction, the result is proved.

This theorem provides us with two criteria for a strong marked digraph to be consistent. Since they involve diwalks, as does the definition of consistency, both are quite similar in nature to the definition.

Balance in signed graphs and digraphs, as well as consistency in graphs, has previously been defined in terms of all cycles (or semicycles) being positive. Our definition seems closer to the objects under investigation here: communication networks with two-version stories being consistently reported. Theorem 1 shows that in the strongly connected case, the two conditions are equivalent.

Our next criterion for consistency is in terms of partitions of the set of nodes and applies to all digraphs, strong or not. IIence this characterization resembles the Structure Theorem for Balance.

Theorem 2. A marked digraph $M$ is consistent if and only if its set of nodes can be partitioned into two sets $V_{0}$ and $V_{1}$ (one possibly empty) with the property that for each set, all arcs from positive nodes go to nodes within the same set and all arcs from negative nodes go to nodes in the other set.

Before proving this result, we illustrate it with the marked digraph $M$ of Fig. 3. The nodes have been labeled so that each $u_{i}$ is positive and each $v_{i}$ negative. That the structure

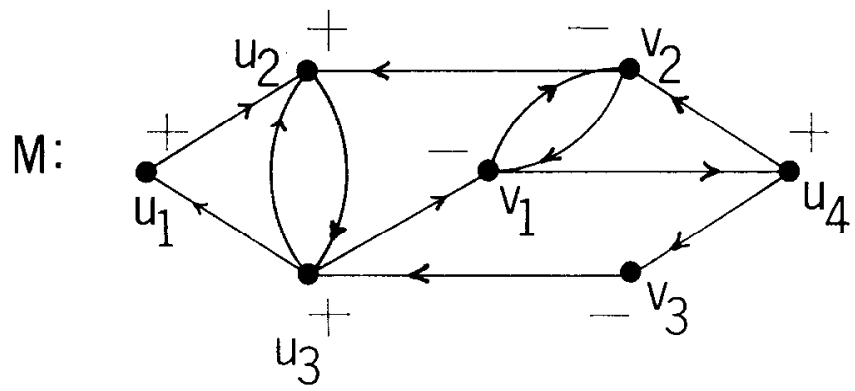

FIG. 3. A consistent marked digraph illustrating the theorem.

is consistent can be seen by observing that any cycle containing one negative node contains exactly two. The two sets described in the theorem can be taken as $V_{0}=\left\{u_{1}, u_{2}\right.$, $\left.u_{3}, v_{1}\right\}$ and $V_{1}=\left\{v_{2}, v_{3}, u_{4}\right\}$. Each node $u_{i}$ then has all its outgoing arcs directed to nodes within the set containing it, and each node $v_{i}$ has all outgoing arcs directed to nodes in the other set.

Proof. In proving the sufficiency of the stated condition, we assume that $M$ is a marked digraph and that $V_{0}$ and $V_{1}$ form a partition of its nodes with the property described in the statement of the theorem. Let $u$ and $v$ be any two nodes. If they are in the same set, then all diwalks from $u$ to $v$ must go between $V_{0}$ and $V_{1}$ an even number of times, so 
that they are all positive; while if $u$ and $v$ are in different sets, all $u: v$ diwalks must similarly be negative. Hence, $M$ must be consistent.

For the converse, assume $M$ is a consistent marked digraph. We prescribe a partition of the nodes of $M$ into two sets $V_{0}$ and $V_{1}$ as follows. Choose an arbitrary node $v_{0}$ and put it into $V_{0}$. For any other node $v$, choose a $v_{0}: v$ path $P$. If $P$ is negative (recall that the sign of path $P$ ignores the sign of $v$ ), put $v$ into $V_{1}$; otherwise into $V_{0}$. (By the definition of consistency, this partition does not depend upon the choice of path.) Suppose that this partition does not satisfy the stated conditions; that is, there is an arc $u v$ such that either (i) $u$ is negative and $u$ and $v$ are in the same set, or (ii) $u$ is positive and $u$ and $v$ are in different sets. In either case, it follows that there are two $v_{0}: v$ diwalks having different signs. This contradicts the hypothesis of consistency and completes the proof.

We observe that as a conscquence of this theorem we have the fact that a consistent marked digraph has no three mutually adjacent negative nodes. This fact also follows readily from the definition since either a cyclic triple of negative nodes or a transitive triple violates the definition.

The directional dual of this theorem is also of some interest and requires no further proof.

THEOREM 2'. A strong marked digraph $M$ is consistent if and only if its set of nodes can be partitoned into two subsets $V_{0}$ and $V_{1}$ (one possibly empty) with the property that for each set, all arcs to positive nodes come from within the same set and all arcs to negative nodes come from outside the set.

This gives a different partition for the consistent net in Fig. 3. Now one set is $\left\{u_{1}, u_{2}\right.$, $\left.u_{3}, v_{2}, v_{3}\right\}$ and the other is $\left\{v_{1}, u_{4}\right\}$.

\section{Consistency and Balance}

Consistency is in fact more closely related to balance than just having a similar definition. The connection is given in the following result. The simple construction given there is illustrated in Fig. 4.

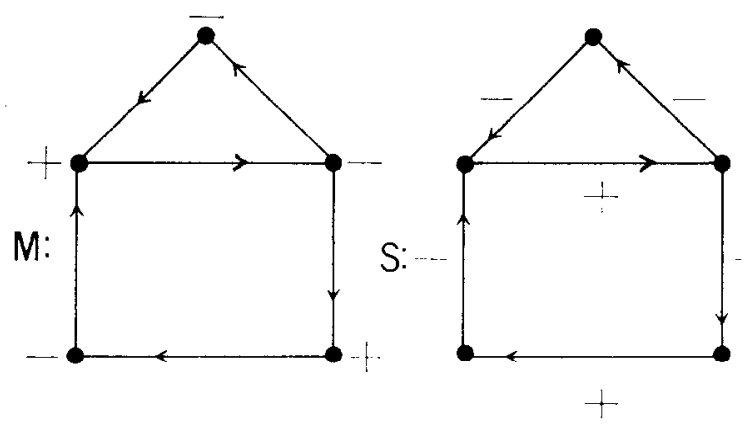

FIG. 4. A marked digraph and its associated signed digraph. 
THEOREM 3. Let $M$ be a strongly connected marked digraph and let $S$ be the corresponding signed digraph in which each arc has the sign of its first node. Then $M$ is consistent if and only if $S$ is balanced.

Proof. Let $M$ be a strong marked digraph and $S$ the corrcsponding signed digraph. Clearly every dicycle of $M$ is positive if and only if every dicycle of $S$ is. Therefore, by Theorem $1, M$ is consistent if and only if $S$ is balanced.

We note that Theorem 3 does not mean that "consistency" and "balance" are equivalent for all strong digraphs. For, the only signed digraphs which are obtained through markings as described in the theorem are those in which all outgoing arcs at each node have the same sign. In terms of this being a "likes-dislikes" model in balance theory, each person either likes no one or dislikes no one.

By directional duality, one also obtains those digraphs in which all incoming arcs are alike at each node, with each person liked by no one or disliked by no one.

It has been shown (Harary, et al., 1965) that in a strongly connected signed digraph, every undirected cycle (semicycle) is positive if every directed cycle (dicycle) is. The corresponding result does not however hold for marked digraphs. The marked digraph $M$ in Fig. 4 is consistent and strongly connected but has a negative undirected cycle. In contrast, all undirected cycles in the corresponding signed digraph are positive.

As tests for consistency of marked digraphs, the criteria of the three theorems vary in the ease of their application. The second theorem probably gives the easiest means of checking, and it can be adapted to all marked digraphs, not just the strongly connected oncs. The algorithm we now give is essentially that given in the proof. First, partition the nodes into two sets $V_{0}$ and $V_{1}$ as follows: Choose an arbitrary node $v$ for $V_{0}$. For any other node $w$, find a $v: w$ path. If it is negative, put $w$ in $V_{1}$, otherwise in $V_{0}$. Now check whether all outgoing arcs from negative nodes go to the other set and all arcs from positive nodes remain within a set. If this is the case, the marked digraph is consistent; if not, it is inconsistent. There are variations of this in which one can check for inconsistencies as one proceeds: Put node $v$ into $V_{0}$. Now take a node $x$ adjacent either (i) from or (ii) to an assigned node $w$. If (i), put $x$ into the set containing $w$ if $w$ is positive and intu the other set if $w$ is negative; if (ii) put $x$ into the set containing $w$ if $x$ is positive and into the other set if $x$ is negative. Before proceeding to another node, check for inconsistencies. This procedure works for all marked digraphs. We illustrate it with the example in Fig. 5. We begin with $v$ in $V_{0}$. Then place $w$ and $x$ in $V_{1}$ since $v$ is negative

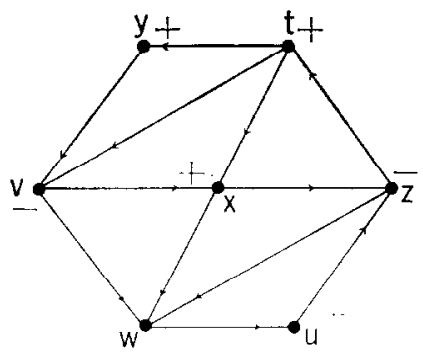

FIG. 5. Testing a marked digraph for consistency. 
and adjacent to both. Now since $w$ is negative and adjacent to $u$, put $u$ in $V_{0}$. Next we take $z$; it is adjacent from $u$ and $x$, both of which are positive and in different sets. This is an inconsistency.

\section{Consistent Marked Tournaments}

An interesting special case of digraphs are the tournaments, those which have exactly one arc between each pair of nodes. (In terms of communication networks, exactly one of each pair would talk to the other.) The following theorem tells which strong marked tournaments are consistent.

THEOREM 4. A strong marked tournament is consistent if and only if it has exactly two negative nodes $u$ and $v$, with arcs from $u$ to all nodes except $v$ and arcs to $v$ from all nodes except $u$.

A non-strong marked tournament is consistent if and only if it can be obtained from such a strong one by reversing arc $u v$, or deleting node $u$, node $v$ or both.

Proof. Clearly any tournament given in the statement of the theorem is consistent. We must show these are the only ones, so we assume $T$ is a consistent marked tournament. By an earlier observation, since any pair of nodes are adjacent, $T$ cannot have three or more negative nodes. Two negative nodes cannot both dominate or both be dominated by a third node, nor can a negative node be on a cyclic triple with two positive ones. Therefore $T$ must be one of the structures described.

'l'he structure of a consistent strong marked tournament $T$ with $p$ points as described in the theorem is shown in Fig. 6. The encircled plus sign indicates an arbitrary subtournament with $p-2$ positive nodes.

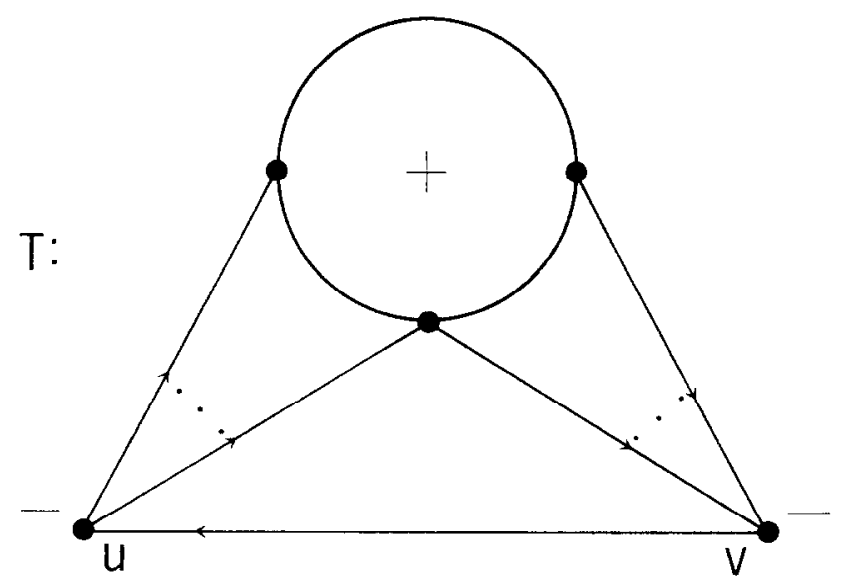

FIG. 6. The structure of a strong marked tournament. 


\section{Consistently Markable Digrayhs}

Thus far we have been considering the question of which marked digraphs are consistent. A related question is: Which digraphs have consistent markings with at least one negative node? We call such digraphs markable. Clearly, any digraph can be marked consistently if all nodes are allowed to be positive, so we do not consider that possibility. Further, if a digraph is bipartite (that is, the nodes can be partitioned into two sets so that all arcs have one point in each set) then it can be consistently marked with all nodes negative. And, of course, the converse is also true: If a consistent marked digraph has all nodes negative it is bipartite. The following consequences of Theorem 2 give a criterion for a digraph to be markable.

THEOREM 5. A digraph $D$ is markable if and only if there is a nonempty proper subset $V_{0}$ of its nodes with the property that for each node $v$, either all of the nodes with arcs from $v$ are in $V_{0}$, or none of them are.

Proof. If $M$ is a consistent marked digraph, let $V_{0}$ be the set described in Theorem 2 . This set has the described property.

Now, assume such a set $V_{0}$ exists. We describe a marking which will be consistent. Let $V_{1}$ be the complement of $V_{0}$. For any node $v$, take $v$ positive if all outgoing arcs stay in the same set $V_{0}$ or $V_{1}$ as $v$, and negative otherwise. It follows from Theorem 2 that the resulting marking is consistent.

We illustrate this construction with the digraph in Fig. 7. If $V_{0}$ is taken as $\left\{v_{2}, v_{3}, v_{5}\right\}$, then for each $v_{i}$ in the digraph either all the nodes or none of the nodes with arcs from $v_{i}$

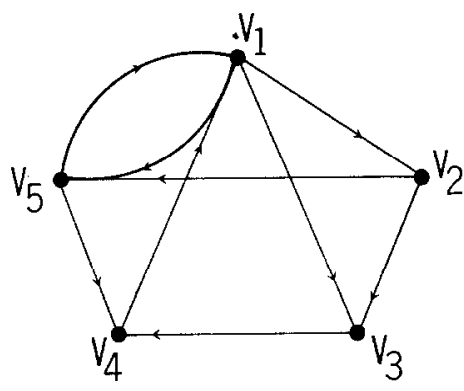

FIG. 7. A markable digraph.

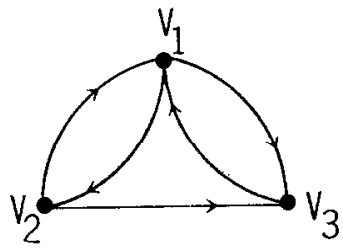

FIG. 8. A nonmarkable digraph.

are in $V_{0}$. Therefore, if $v_{2}$ and $v_{4}$ are taken as positive and $v_{1}, v_{3}$, and $v_{5}$ as negative, then the resulting marked digraph is consistent. An example of a digraph which is not markable is given in Fig. 8. The adjacencies dictate that $v_{1}$ and $v_{2}$ must both be in $V_{0}$ or $V_{1}$, as must $v_{1}$ and $v_{3}$. Therefore, by Theorem 5 , there is no set $V_{0}$ as required for a consistent marking. 


\section{Concluding Comments}

In subsequent work, we plan to investigate marked (undirected) graphs. These are apparently more difficult, as no complete characterization of consistency is known. One might ask why, if digraphs are more general than graphs, can the theorems for directed graphs not be applied to the undirected case. The answer lies in the dicycles of length 2 . The usual way of convcrting a graph to a digraph is to rcplacc each undirected edge by a symmetric pair of arcs, one in each direction, as in Fig. 9. While this does in fact preserve

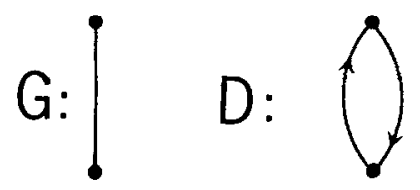

FIG. 9. Forming the digraph of a graph.

most graphical properties, negative dicycles are introduced for each edge joining two nodes of opposite sign. Hence, this procedure is of no value in studying consistency, and the two cases, directed and undirected, are quite different.

Related work is given by Harary, Palmer, Robinson, and Schwenk (1977). This is the development of a series of formulas, using techniques of combinatorial enumeration, to determine the number of isomorphism classes of each of the following types of configurations with a given number of nodes:

(1) signed graphs (and digraphs),

(2) marked graphs,

(3) graphs with both signed points and lines.

Further, the number of configurations is also determined when an interchange of the signs of the points and/or the lines is also regarded as an equivalence relation.

\section{REFERENCES}

Cartwright, D., \& Harary, F. Structural balance: A generalization of Heider's theory. Psychological Review, 1956, 63, 277-293.

Cartwright, D., \& Harary, F. Balance and clusterability: An overview. In P. Holland \& S. Leinhardt (Eds.), Social networks: Surveys, advances, and commentaries. New York: Academic Press, 1976.

HARARY, F. On the notion of balance in a signcd graph. Michigan Mathematical Journal, 1953, 2, 143-146.

Harary, F. Structural duality. Behavioral Science, 1957, 2, 255-265.

Harary, F. On the measurement of structural balance. Beharioral Science, 1959, 4, 316-323.

Harary, F., Norman, R., \& Cartwright, D. Structural models: An introduction to the theory of directed graphs. New York: Wiley, 1965.

Harary, F., Palmer, E. M., Robinson, R. W., \& Schwenk, A. J. Enumeration of graphs with signed points and lines. Journal of Graph Theory, 1977, 1, 295-308. 
HeIDER, F. Attitudes and cognitive organization. Journal of Psychology, 1946, 21, 107-112.

Oscood, C. E., \& Tannenbaum, P. H. The principle of congruity in the prediction of attitude change. Psychological Review, 1955, 62, 42-55.

Zajonc, R. B. Cognitive theories of social psychology. In G. Lindzey \& E. Aronson (Eds.), The handbook of social psychology, Vol. 1. Reading, Mass.: Addison-Wesley, 1968. Pp. 320-411.

ReCEIVED: April 25, 1978 